\title{
OS FUNGOS ANEMÓFILOS E SENSIBILIZAÇÃO EM INDIVÍDUOS ATÓPICOS EM PORTO ALEGRE, RS
}

\author{
Adelina Mezzari*, Christiano Perin, Sidnei Alves Santos Júnior, luiz Antonio Guerra Bernd, Giovani Di Gesu \\ Trabalho realizado na Fundação Faculdade Federal de Ciências Médicas de Porto Alegre - Faculdade de \\ Farmácia da Universidade Federal do Rio Grande do Sul - Curso de Pós-graduação em Ciências Veterinárias da \\ Universidade Federal do Rio Grande do Sul, Porto Alegre, RS.
}

RESUMO - Oв|ETIVos. Identificar e caracterizar a freqüência e a concentração de esporos de fungos anemófilos em Porto Alegre. Determinar a prevalência de sensibilização a estes fungos em indivíduos atópicos.

Métodos. Em 52 amostras colhidas no equipamento Rotorod Sampler ${ }^{\circledR}$, foram quantificados 3773 esporos de fungos no período de um ano (abril de 2000 a março de 200I). Em 39 pacientes atópicos com rinite e ou asma foram realizados testes cutâneos com alérgenos de fungos anemófilos e $o$ teste fluorimunoenzimático para verificar a incidência de manifestações alérgicas provocadas por tais fungos.

Resultados. Em 3773 esporos de fungos encontrados foram identificados 674 (17,86\%) de Cladosporium sp, 567 (15,03\%) do grupo Aspergillus/Penicillium, 144 (3,82\%) de rusts, 94 (2,49\%) de Helminthosporium sp, 46 (I,22\%) de Botrytis sp, 45
(I,I9\%) de Alternaria sp, $34(0,90 \%)$ de smuts, $33(0,87 \%)$ de Curvularia sp, $23(0,61 \%)$ de Nigrospora sp e $3(0,08 \%)$ de Fusarium sp. Também foram encontrados $1905(50,49 \%)$ de ascosporos e 145 (3,84\%) de basidiosporos. Não foram possíveis de serem identificados 60 (I,59\%) dos esporos observados. Nos 39 testes cutâneos e sorológicos realizados, resultaram em $15,38 \%$ de indivíduos atópicos sensibilizados por alérgenos de fungos em Porto Alegre.

Conclusōes. A ocorrência de grande número de esporos de fungos no ar em nossa cidade enfatiza a importância deste estudo sobre os fungos anemófilos, sendo eles responsáveis por $15,38 \%$ das sensibilizações alérgicas em indivíduos atópicos com manifestações de asma e ou rinite.

UnItermos: Fungos anemófilos. Asma. Rinite. Sensibilização.

\section{INTRODUÇÃO}

Os fungos dispersam-se na natureza através do ar atmosférico ou por outras vias, como água, insetos, homem e animais. Os fungos que são dispersados através do ar atmosférico são denominados fungos anemófilos. Sendo assim, a microbiota fúngica anemófila pode ser semelhante ou diferente em cada cidade ou região. Os elementos fúngicos que são encontrados no ar atmosférico são os esporos (propágulos). São aeroalérgenos que, quando inalados, podem ser responsáveis por manifestações respiratórias alérgicas, como asma e rinite'.

O homem, ao se expor inalando estes aeroalérgenos, pode desenvolver uma doença respiratória alérgica e a intensidade de exposições pode determinar a relevância clínica. Para controlar as manifesta-

* Correspondência:

Fundação Faculdade Federal de

Ciências Médicas de Porto Alegre

Rua Sarmento Leite, 245

90050-170, Porto Alegre, RS ções alérgicas provocadas por estes alérgenos inalantes através da terapêutica específica, é importante conhecer a freqüência com que ocorre determinado fungo anemófilo, em relação ao total de exposições praticadas pelo indivíduo ou do número de amostras isoladas ${ }^{2}$. Segundo Horner et al. (1995) ${ }^{4}$, nos Estados Unidos e em outros países industrializados, cerca de $20 \%$ da população apresenta doenças alérgicas como asma e ou rinite causadas por aeroalérgenos de fungos.

Para caracterizar a composição de fungos no ar atmosférico, atualmente, estão disponíveis equipamentos que utilizam amostra volumétrica de ar. Estes equipamentos permitem definir a periodicidade destes fungos anemófilos. Porém, éimportante ressaltar que existe diferença entre os fungos predominantes no ambiente domiciliar e no ar atmosférico extradomiciliar4.

Apesar de os fungos terem reconhecida participação em quadros de hipersensibilidade do trato respiratório, as publicações sobre a presença de fungos na atmosfera das cidades brasileiras são reduzidas ${ }^{6,9}$. Com isso, atualmente, há grande dificuldade na caracterização da importância dos alérgenos de fungos em quadros de asma e rinite alérgica. Em parte, isto se deve ao desconhecimento da microbiota fúngica a que a população está exposta. Desta forma a identificação equantificação dos esporos de fungos anemófilos na cidade, bem como sua aplicação na clínica de alergias, poderá permitir avanços no diagnóstico e desenvolvimento de novos métodos de abordagem nestas patologias ${ }^{2}$.

No presente trabalho, pesquisamos a quantidade de fungos anemófilos extradomiciliares presentes em Porto Alegre, durante o período de um ano (abril de 2000 a março de 200I). Utilizamos o equipamento Rotorod Sampler $40 \circledR$. Posteriormente, investigamosa incidência das manifestações alérgicas de rinite e ou asma por fungos em indivíduos atópicose sadios (grupo controle). Utilizamos testes cutâneos em todos os indivíduos e sorológicos nos indivíduos atópicos.

O objetivo fundamental deste estudo foi identificar e caracterizar a freqüência e a 
concentração de esporos de fungos anemófilos em Porto Alegre. Determinar a prevalência de sensibilização a estes fungos em indivíduos atópicos.

\section{Métodos}

Os materiais e métodos constam de dois estudos:

Pesquisa dos fungos anemófilos: $O$ presente trabalho foi realizado usando o coletor aeroscópico Rotorod Sampler ${ }^{\circledR}$ modelo 40, para a pesquisa e quantificação dos fungos anemófilos ${ }^{10}$. O equipamento foi colocado no topo do edifício de cinco andares da Fundação Faculdade Federal de Ciências Médicas de Porto Alegre, a uma altura de 23 metros do solo. As coletas foram realizadas durante 24 horas, uma vez por semana, durante o período de um ano (abril de 2000 a março de 200I). Este equipamento é constituído de um coletor de impacto para a coleta de partículas na superfície de dois bastões (haste) com dimensões de I,52 mmX I,52 mm X $32 \mathrm{~mm}$. Estas hastes estão fixadas por um prendedor de metal de aproximadamente $10 \mathrm{~cm}$ de comprimento, conectado a um pequeno motor. $\mathrm{A}$ ação do motor faz com que $\circ$ bastão gire rapidamente. As partículas suspensas na atmosfera são recolhidas por este coletor. Posteriormente, o bastão foi retirado e sobre ele foi sobreposta uma lamínula $(22 \mathrm{~mm} \mathrm{X}$ $22 \mathrm{~mm}$ ). A leitura foi realizada no microscópio óptico onde foi feita a identificação e a quantificação dos esporos fúngicos, mantendo o padrão definido pelo fabricante. Segundo o padrão, a quantificação corresponde ao número total de esporos dos fungos observados em toda a extensão da lamínula. Posteriormente, este número é calculado pelo volume de ar do período de tempo definido pelo ciclo utilizado. Utilizamos o padrão do fabricante definido como um ciclo de $10 \%$ do total, em 24 horas de coleta. Neste ciclo, o volume constante medido foi de $3,12 \mathrm{~m}^{3}$ de ar. O resultado final foi calculado pelo número de esporos de fungos contados no bastão coletor e divididos pelo volume padrão de $3,12 \mathrm{~m}^{3} \mathrm{de}$ ar, resultando na quantidade de esporos por $\mathrm{m}^{3} \mathrm{de}$ ar em 24 horas. Durante o período avaliado, foram realizados 52 coletas, resultando em 3773 esporos de fungos.

Durante o período estudado, foram determinadas as temperaturas máximas e mínimas, a umidade relativa do ar e a precipitação atmosférica durante as 24 horas da coleta. Estes dados foram fornecidos pelo Instituto Nacional de Metereologia do Oitavo Distrito de Metereologia (INMET), do Ministério da Agricultura, Pecuária e Abastecimento (MAPA).

Paraa identificação dos esporos dos fungos anemófilos foi adotada a chave sistemática da American Academy of Allergy Asthma \& Immunology (AAAAl, 1997)". Na identificação dos esporos de fungos, a partir da coleta quantitativa em equipamentos coletores, alguns esporos de fungos apresentam morfologia semelhante, tornando sua identificação inviável em nível de gênero, como pode ocorrer, por exemplo, com oAspergillussp e Penicillium sp. Outros esporos, como os ascosporos e basidiosporos podem não ser identificados como partículas específicas por pertencerem ao filo Fungi Imperfect, no qual as formas liberadas nem sempre apresentam características individuais capazes de serem identificadas.

Determinação da sensibilização alérgica: Existe dificuldade na padronização de alérgenos de fungos para aplicação diagnóstica' ${ }^{2}$. Isto ocorre porque os fungos apresentam variabilidade em sua composição bioquímicae imunológica. Nos últimos anos, surgiram preparações padronizadas, utilizando antígenos purificados e específicos de cada espécie fúngica, visando obter resultados mais específicos ${ }^{6}$. Pesquisamos a sensibilização alérgica em indivíduos atópicos e em um grupo-controle de indivíduos sadios que não apresentavam asma e ou rinite, segundo informações dos mesmos. Todos foram submetidos a testes cutâneos de leitura imediata com os extratos alergênicos preparados pela IPI-ASAC Brasil. Foram utilizados os alérgenos dos fungos Aspergillus fumigatus, Penicillium notatum, Cladosporium herbarum e Alternaria alternata e dos ácaros domésticos Dermatophagoides pteronyssinus, Dermatophagoides farinae e Blomia tropicalis. No mesmo momento, foram colhidas amostras de sangue dos indivíduos atópicos para determinação de anticorpos lgE total e específica para os alérgenos dos fungos Aspergillus niger, Aspergillus fumigatus, Penicillium notatum, Cladosporium herbarum e Helminthosporium halodes. O teste cutâneo foi aplicado na face volar do antebraço, na qual colocou-se uma gota de cada alérgeno, deixando entre os mesmos um espaço mínimo de $2 \mathrm{~cm}$. Através da gota foi passado o bisel de uma agulha hipodérmica descartável $(13 \mathrm{mmX}$ $0,45 \mathrm{~mm}-26 \mathrm{GX} 1 / 2$ ) e executada uma leve ruptura de pequena camada da pele, evitandose o sangramento. Após um minuto aproximadamente, foi retirado o excesso de líquido com papel absorvente. Procedimento idêntico foi efetuado com o controle positivo da reação (solução de histamina glicerinada fenolada) e com o controle negativo (solução salina glicerinada fenolada).

A leitura foi realizada após 15 minutos, observando-se a formação de pápula e eritema. O valor de referência para a reação positiva é de $2 \mathrm{~mm}$ a $3 \mathrm{~mm}$ de diâmetro de pápula. Neste estudo, considerou-se reação positiva a formação de pápula ou eritema com $2 \mathrm{~mm}$ ou mais de diâmetro.

A pesquisa de lgE total e específica foi realizada utilizando 0 teste fluorimunoenzimático no sistema CAP (Pharmacia \& Upjohn). Os resultados são referidos como ausência ou presença de IgE específica para o alérgeno na amostra analisada. Os níveis deste anticorpo são expressos em unidade internacional por mililitro $(\mathrm{UI} / \mathrm{mL})$, segundo os valores de referência e expressos como: Ausência de IgE específica para o alérgeno quando $<0,35$ $\mathrm{Ul} / \mathrm{mL}=$ classe 0 ou: Presença de lgE específica entre $0,35 \mathrm{UI} / \mathrm{mL}$ e $0,70 \mathrm{Ul} / \mathrm{mL}=$ classe $\mathrm{l}$; entre $0,70 \mathrm{Ul} / \mathrm{mL}$ e $3,50 \mathrm{UI} / \mathrm{mL}=$ classe $I$; entre $3,50 \mathrm{UI} / \mathrm{mL}$ e $17,5 \mathrm{UI} / \mathrm{mL}=$ classe III e $>17,50 \mathrm{UI} / \mathrm{mL}=$ classe IV.

Foram selecionados pacientes com asmae ou rinite alérgica, de todas as idades e ambos os sexos, do ambulatório de Alergia e Imunologia Clínica da Fundação Faculdade Federal de Ciências Médicas de Porto Alegre naSanta Casa de Misericórdia de Porto Alegre.

Os indivíduos que participaram dos testes não utilizaram anti-histamínicos, corticosteróides sistêmicos ou antidepressivos tricíclicos, nos sete dias que precederam a realização dos testes. Antes do teste, o paciente forneceu as informações e assinou um termo de consentimento.

\section{Resultados}

Fungos anemófilos: Das 52 amostras coletadas foram quantificados 3773 esporos de fungos anemófilos durante 0 período pesquisado (abril de 2000 a março de 200I) em 
Porto Alegre. Os esporos de fungos encontrados foram 674 (17,86\%) de Cladosporium sp; 567 (I5,03\%) do grupo Aspergillus/Penicillium; 144 (3,82\%) de rusts; 94 (2,49\%) de Helminthosporium sp; 46 (I,22\%) de Botrytis sp; 45 (I,19\%) de Alternaria sp; 34 (0,90\%) de smuts; 33 (0,87\%) de Curvularia sp; 23 (0,61\%) de Nigrospora sp e $3(0,08 \%)$ de Fusarium sp. Também foram encontrados 1905(50,49\%) de ascosporos e 145 (3,84\%) de basidiosporos. Não foram possíveis de serem identificados 60 (I,59\%) dos esporos observados.

Durante o período estudado, a média mínimae máxima da temperatura foi de $8^{\circ} \mathrm{Ce} 27^{\circ} \mathrm{C}$, da umidade relativa do ar foi de $62 \%$ e $87 \%$ eda pressão atmosférica foi de 1012 hectopascaise 1022 hectopascais, respectivamente.

\section{Pesquisa imunológica}

a)Testes cutâneos: Foram realizados 39 testes cutâneos em indivíduos atópicos. Destes, quatro $(10,26 \%)$ foram positivos para 0 alérgeno Aspergillus fumigatus, um (2,56\%) Penicillium notatum, dois $(5,12 \%)$ Cladosporium herbarum e um $(2,56 \%)$ Alternaria alternata. Um individuo foi positivo para os alérgenos de Aspergillus, Penicillium e Cladosporium ao mesmo tempo. Totalizando seis (15,38\%) indivíduos sensibilizados para os alérgenos de fungos em nossa cidade, conforme Tabela I.

Entre os 31 indivíduos sadios do grupocontrole, não houve reação positiva a nenhum alérgeno dos fungos testados, Alternaria alternata, Aspergillus fumigatus, Cladosporium herbarum e Penicillium notatum. Destes indivíduos, nove $(29,03 \%)$ foram positivos para pelo menos um alérgeno dos ácaros domésticos testados, Dermatophagoides pteronyssinus, Dermatophagoides farinae e Blomia tropicalis.

b) Pesquisa IgE total e específica: Foram utilizadas 39 amostras de sangue colhidas dos indivíduos atópicos que realizaram os testes cutâneos. Os resultados obtidos foram equivalentes aos testes cutâneos nos quais seis (I5,38\%) dos indivíduos testados foram positivos para os alérgenos testados. Destes, um $(2,56 \%)$ foi positivo para os alérgenos de Aspergillus fumigatus, Aspergillus niger, Penicillium notatum, Cladosporium herbarum e Helminthosporium halodes e dois $(5,12 \%)$ foram positivos para o Cladosporium herbarum e Helminthosporium halodes ao mesmo tempo conforme Tabela 2.

\begin{tabular}{lcccr}
\hline \multicolumn{5}{c}{ Tabela I - Testes cutâneos realizados em 39 indivíduos atópicos } \\
\hline Alérgeno & Alternaria & Aspergillus & Cladosporium & Penicillium \\
& alternata & fumigatus & herbarum & notatum \\
Positivo & $\mathrm{I}$ & $4^{*}$ & $2 *$ & $2^{*}$ \\
Negativo & 38 & 35 & 37 & 38 \\
\hline
\end{tabular}

* Reação positiva no mesmo indivíduo

\begin{tabular}{|c|c|c|c|c|c|}
\hline Alérgeno & $\begin{array}{l}\text { Aspergillus } \\
\text { fumigatus }\end{array}$ & $\begin{array}{l}\text { Aspergillus } \\
\text { niger }\end{array}$ & $\begin{array}{c}\text { Cladosporium } \\
\text { herbarum }\end{array}$ & $\begin{array}{l}\text { Helminthos } \\
\text { halodes }\end{array}$ & $\begin{array}{c}\text { Penicillium } \\
\text { notatum }\end{array}$ \\
\hline Positivo & $3^{*}$ & |* & $3 *$ **⿻丷木 & $3 * * *$ & I* \\
\hline Negativo & 37 & 38 & 35 & 35 & 38 \\
\hline
\end{tabular}

Tabela 3 - Comparação entre os resultados dos testes cutâneos e sorológicos

\begin{tabular}{lrrrrrr} 
& \multicolumn{5}{c}{ Resultado do teste sorológico } \\
\cline { 2 - 7 } Resultado do & \multicolumn{1}{c}{ Negativo } & \multicolumn{2}{c}{ Positivo } & \multicolumn{2}{c}{ Total } \\
Teste cutâneo & $n$ & $\%$ & $n$ & $\%$ & $n$ & $\%$ \\
Negativo & 30 & 76,9 & 2 & 5,1 & 32 & 82,1 \\
Positivo & 4 & 10,3 & 3 & 7,7 & 7 & 17,9 \\
Total & 34 & 87,2 & 5 & 12,8 & 39 & 100,0 \\
\hline
\end{tabular}

Coeficientede concordância Kappa $=0,412$

Para comparar os resultados dos testes cutâneos e sorológicos (positivos ou negativos), foi construída uma tabela cruzada, no qual se verifica que háuma concordância nos resultados dos 39 pacientes investigados, conforme Tabela 3. Para quantificara concordânciaentreos resultados dos doistestes, recorreu-seao coeficiente de $\mathrm{Kappa}^{3}$. Trata-se de uma medida no intervalo de 0 a I que medeograu de concordância entre duas classificaçõesorganizadas naforma de uma tabela cruzada simétrica. Nesse caso, 0 coeficiente resultou em 0,4I2, considerado "moderado" segundo Landis \& Kock (1977) ${ }^{7}$.

$O$ banco de dados foi organizado no Microsoft Excel e a Análise estatística foi realizada no SPSS versão 8.0. Foram utilizadas técnicas de estatística descritiva como tabelase freqüência, medidas de tendência central e de variabilidade e o coeficiente de concordância de Kappa. No campo referencial foram construídos intervalos de confiança para prevalência. Não foram utilizados testes de hipóteses, mas sim intervalos de confiança.

\section{Discussão}

As freqüências de $17,86 \%$ de Cladosporium sp, $15,03 \%$ do grupo Aspergillus/ Penicillium, 3,82\% de rusts, 2,49\% Helminthosporium sp, I,22\% Botrytis sp, I, 19\% Alternaria sp, 0,90\% smuts, 0,87\% Curvularia sp, $0,61 \%$ Nigrospora sp, $0,08 \%$ Fusarium sp, $50,49 \%$ de ascosporos e $3,84 \%$ de basidiosporos nos induziram a realizar os testes cutâneos em indivíduos atópicos com os extratos alergênicos de Aspergillus fumigatus, Penicillium notatum, Cladosporium herbarum e Alternaria alternata e a determinação de lgE específica para os fungos Aspergillus fumigatus, Aspergillus niger, Penicillium notatum, Cladosporium herbarum e Helminthosporium halodes disponíveis em nosso meio para uso.

Os esporos rusts e smuts, presentes em nosso trabalho, são patógenos de plantas e também alérgenos do homem. Aidentificação de esporos de fungos é tarefa difícil, uma vez que alguns fungos, por terem formas similares, não possibilitam a identificação em nível de 
gênero, caso do Aspergillus e do Penicillium. Outros esporos são muito pequenos e suas características individuais, como a transparência, impedem a identificação de gênero, a exemplo dos ascosporos e basidiosporos

Ao avaliarmos a pesquisa imunológica com o teste cutâneo e a pesquisa de lgE específica para os fungos anemófilos presentes em nossa cidade, observamos que dos 39 testes realizados, 6 (I5,38\%) indivíduos atópicos estão sensibilizados por estes fungos anemófilos. Este resultado revela o risco maior que estes indivíduos têm de apresentar crises alérgicas de asma e ou rinite quando expostos a estes alérgenos. Um indivíduo foi positivo para todos os alérgenos testados. Em dois indivíduos ocorreu reação positiva para os alérgenos de Cladosporium herbarum e Helminthosporium halodes, ao mesmo tempo, porém o alérgeno Helminthosporium halodes não foi utilizado nos testes cutâneos ${ }^{4}$.

Estudos similares com pesquisa cutânea em pacientes com alergia respiratória ${ }^{5,8}$ foram realizados em São Paulo. Gambale et al. $(1993)^{5}$ investigaram em livrarias da Universidade de São Paulo os fungos do ar ali presentes e sua relação com a alergia respiratória nos indivíduos que ali trabalhavam, encontrando 19,5\% dos sintomáticos e $9 \%$ dos assintomáticos com reação positiva aos fungos do ar presentes nesse ambiente. Mohovic et al. $(1988)^{8}$ investigaram a positividade cutânea em pacientes com alergia respiratória utilizando 42 extratos alergênicos de fungos anemófilos encontrados na cidade de São Paulo e obtiveram $34,8 \%$ de reação positiva ao utilizar um extrato polivalente destes fungos e, quando submetidos ao extrato individual de cada fungo, $74,3 \%$ desses pacientes foram positivos para um ou mais extratos alergênicos.

\section{Conclusão}

A ocorrência de grande número de esporos de fungos nas diversas coletas efetuadas, enfatiza a importância deste estudo sobre os fungos anemófilos em nossa cidadee também em nosso país.
Nosso estudo foi o primeiro passo para padronizarmos e conhecermos a flora anemófila de Porto Alegre. Neste estudo, 15,38\% dos indivíduos atópicos com asma e ou rinite em Porto Alegre são sensibilizados por fungos anemófilos. Este resultado é equivalente ao que ocorre nos Estados Unidos e outros países industrializados onde cerca de $20 \%$ da população apresentam doenças respiratórias alérgicas como asma e rinite causadas por aeroalérgenos de fungos.

\section{SUMMARY}

Airborne fung and SEnSitization IN atopic individuals in Porto Alegre, RS, BrAZIL

OBJECTIVE. To identify and quantify the fungal spores in the atmospheric air of Porto Alegre and to assess sensitization to these allergens in atopic individuals suffering from rhinitis and asthma.

Methods. A total of 52 samples were obtained using a Rotorod Sampler ${ }^{\circledR}$ from April 2000 through March 2000I. Allergy skin tests with anemophilous fungus allergens as well as fluorimmunoenzymatic tests were performed in 39 atopic individuals with rhinitis and asthma in order to detect the incidence of respiratory allergies caused by these airborne fungi.

RESULTS. In the 3773 fungal spores detected in this study, there was a prevalence of ascosporos (50.49\%), Cladosporium (I7.86\%), Aspergillus/Penicillium (15.03\%), basidiosporos (3.84\%), rusts (3.82\%), and Helminthosporium (2.49\%), as well as a lesser frequency of Botrytis (1.22\%), Alternaria (I.19\%), smuts (0.90\%), Curvularia $(0.87 \%)$, Nigrospora $(0.61 \%)$, and Fusarium $(0.08 \%)$. Sixty spores (1.59\%) detected here could not be identified by the systematic key used. Among the atopic individuals tested, $15.38 \%$ were found to be sensitized by these anemophilous fungi allergens.

ConcLusion. The occurrence of a great number of fungal spores emphasizes the importance of studying anemophilous fungi locally, since they are responsible for $15.38 \%$ of allergic sensitization in atopic individuals with asthma and rhinitis. [Rev Assoc Med Bras 2003; 49(3): 270-3]

KEY wORDs: Airborne fungi. Asthma. Rhinitis. Sensitization

\section{REFERÊNCIAS}

I. Burge HA, Levetin E, Muilenberg, ML, Solomon, WR. Fungus spore identification. American Academy of Allergy Asthma Immunology; 1996. p.3-22.

2. Chapman, JA. How relevant are pollen and mold spore counts to clinical practice? Ann Allergy Asthma Immunol 2000; 84:467-8.

3. Everit, BS. The analysis of contingency tables. 2nd ed. London: Chapman \& Hall; 1992.

4. Horner WE, Helbling A, Salvaggio JE, Lehrer, SB. Fungal allergens. Clin. Microbiol Rev 1995; 8:16I-79.

5. Gambale W, Croce J, Costa-Manso E, Croce, MS. Library fungi at the University of São Paulo and their relationship with respiratory allergy.J Invest Allergol Clin Immunol I 993; 3:45-50.

6. Gompertz OF, GambaleW, Paula CR, Corrêa B. Fungos e alergia. In: Trabulsi LR, Alterthum F, Gompertz OF, Candeias JAN. Microbiologia. 3rd ed. São Paulo: Atheneu; 1999. p.42I-2.

7. Landis JR, Koch GG. The measurements of observer agreement for categorical data. Biometrics 1977; 33:159-74.

8. Mohovic J, Gambale W, Croce, J. Cutaneous positivity in patients with respiratory allergies to 42 allergenic extracts of airborne fungi isolated in São Paulo, Brazil. Allergol Immunopathol (Madr). 1988; 16:397-402.

9. Oliveira Lima A, Seabra O, França AT, Cukier, J. Incidência de fungos na atmosfera de algumas cidades brasileiras. Hospital (Rio de Janeiro) 1963; 63:93-102.

10. Frenz DA. Comparing pollen and spore counts collected with the Rotorod Sampler ${ }^{\circledR}$ and Burkard $\AA$ spore trap. Ann Allergy Asthma Immunol I 999; 83:34I-7.

II. American Academy Allergy Asthma and Immunology (AAAAI). Aeroallergen identification course. AAAl; 1997.

12. Al-DooryY, Domson JF. Mould allergy. Philadelphia: Lea e Fabirger; 1984.

Artigo recebido:03/07/02

Aceito para publicação: 31/0I/03 